\title{
Do Solar Coronal Holes Affect the Properties of Solar Energetic Particle Events?
}

\author{
S.W. Kahler • C.N. Arge • S. Akiyama • N. Gopalswamy
}

Received: 2 November 2012 / Accepted: 4 October 2013 / Published online: 8 November 2013

(C) Springer Science+Business Media Dordrecht (outside the USA) 2013

\begin{abstract}
The intensities and timescales of gradual solar energetic particle (SEP) events at 1 AU may depend not only on the characteristics of shocks driven by coronal mass ejections (CMEs), but also on large-scale coronal and interplanetary structures. It has long been suspected that the presence of coronal holes $(\mathrm{CHs})$ near the CMEs or near the 1-AU magnetic footpoints may be an important factor in SEP events. We used a group of $41 E \approx 20 \mathrm{MeV}$ SEP events with origins near the solar central meridian to search for such effects. First we investigated whether the presence of a $\mathrm{CH}$ directly between the sources of the $\mathrm{CME}$ and of the magnetic connection at $1 \mathrm{AU}$ is an important factor. Then we searched for variations of the SEP events among different solar wind (SW) stream types: slow, fast, and transient. Finally, we considered the separations between $\mathrm{CME}$ sources and $\mathrm{CH}$ footpoint connections from 1 AU determined from four-day forecast maps based on Mount Wilson Observatory and the National Solar Observatory synoptic magnetic-field maps and the Wang-Sheeley-Arge model of SW propagation. The observed in-situ magnetic-field polarities and SW speeds at SEP event onsets tested the forecast accuracies employed to select the best SEP/CH connection events for that analysis. Within our limited sample and the three analytical treatments, we found no statistical evidence for an effect of CHs on SEP event peak intensities, onset
\end{abstract}

Solar Origins of Space Weather and Space Climate

Guest Editors: I. González Hernández, R. Komm, and A. Pevtsov

S.W. Kahler $(\bowtie) \cdot$ C.N. Arge

Air Force Research Laboratory, Space Vehicles Directorate, 3550 Aberdeen Ave., Kirtland AFB, NM 87117, USA

e-mail: stephen.kahler@ kirtland.af.mil

C.N. Arge

e-mail: nick.arge@kirtland.af.mil

S. Akiyama

The Catholic University of America, Washington, DC 20064, USA

e-mail: sachiko.akiyama@nasa.gov

N. Gopalswamy

NASA Goddard Space Flight Center, Greenbelt, MD 20771, USA

e-mail: nat.gopalswamy@nasa.gov 
times, or rise times. The only exception is a possible enhancement of SEP peak intensities in magnetic clouds.

Keywords Energetic particles - acceleration - Magnetic fields - models · Coronal mass ejections - low coronal signatures

\section{Introduction}

\subsection{Coronal Hole Locations and Retarded Solar Energetic Particle Event Onsets}

One of the largest $E>10 \mathrm{MeV}$ solar energetic particle (SEP) events of Solar Cycle 21 began on 6 June 1979 at about 1850 UT. The associated X2 flare peaked at 0516 UT on 5 June, so the time from flare peak to SEP onset at 1 AU was more than 37 hours: a surprisingly long time considering its source location near the central meridian at N20E16 in NOAA Active Region (AR) 1781. The absence of a prompt onset in this SEP event was first noted by von Rosenvinge and Reames (1983), who pointed out the presence of a coronal hole $(\mathrm{CH})$ to the West of AR 1781 and conjectured that SEPs diffusing westward from the AR were intercepted by the open fields of the $\mathrm{CH}$. An associated large, but poorly observed, east-limb CME was reported for this event in the P78-1 Solwind transient list (Howard et al., 1985), but Bravo $(1993,1995)$ cited this event as an example of the view that CMEs with interplanetary shocks were only by-products of fast solar wind (SW) eruptions in adjacent $\mathrm{CHs}$ and were not drivers of the shocks. Although this idea was not accepted by the community, the possibility of some $\mathrm{CH}$ connection to SEP events and CMEs remained. The SEP onset on 6 June also occurred as the Earth moved from a negative to a positive polarity SW sector with its source in the nearby $\mathrm{CH}$. This was the basis of an alternative interpretation by Kahler, Kunches, and Smith (1995), who proposed that the open fields of the adjacent $\mathrm{CH}$ were filled with SEPs, but only up to the interplanetary current sheet, which acted as a barrier to SEP propagation into the negative polarity region. These authors retracted this interpretation when their statistical study (Kahler, Kunches, and Smith, 1996) showed that SEP event properties are independent of whether the SEP source is in the same or a different sector as the observer at $1 \mathrm{AU}$.

A more convincing argument for $\mathrm{CH}$ effects on SEP events was made by Kunches and Zwickl (1999) using the NOAA Space Environment Solar Catalog (SESC) of large E > $10 \mathrm{MeV}$ events and their associated flares. For suitable events, they examined He $10830 \AA$ disk images to determine whether a $\mathrm{CH}$ lay on a line between the flare $\mathrm{AR}$ and the footpoint of Earth's magnetic-field line (hereafter 1 AU footpoint) calculated kinematically from the local SW speed. In nearly all cases with interposed CHs, including the 5 June 1979 flare, the flare AR lay in the eastern hemisphere. Plots of the times from X-ray flare peak to 1 AU SEP onset of the $\approx 30 \mathrm{MeV}$ proton events versus either solar longitude or azimuthal separations of flare AR from $1 \mathrm{AU}$ footpoint yielded a population of generally longer onset times for events with interposed CHs. For the physical explanation of this effect, the authors suggested only that coronal shocks were somehow retarded in their passages through the $\mathrm{CH}$ high-speed streams before reaching the field lines connecting to Earth.

In a more recent test of $\mathrm{CH}$ effects on SEP events, Shen et al. (2006) selected as candidates for SEP production a sample of $56 \mathrm{CMEs}$ with projected speeds and widths exceeding $1000 \mathrm{~km} \mathrm{~s}^{-1}$ and $130^{\circ}$, respectively, and originating from the western hemisphere. These authors found no dependence of $E>10 \mathrm{MeV}$ or $E>50 \mathrm{MeV}$ SEP production on the proximity of CHs, determined from $284 \AA$ solar images, to the CME sources. In the spirit of the Kunches and Zwickl (1999) result, they separated cases with and without CHs extending 
into the longitudes between the CME sources and the $1 \mathrm{AU}$ footpoints, again finding no significant difference in SEP production between the two groups. A subsequent study (Shen et al., 2010) with an updated list of 76 fast and wide western-hemisphere CMEs and CHs, now based on photospheric-field extrapolations, confirmed their earlier result of no effect of $\mathrm{CHs}$ on SEP production.

A problem arises, however, in comparing the apparently conflicting Shen et al. (2010) results with those of Kunches and Zwickl (1999). While Shen et al. (2010) determined that 61 of 76 western-hemisphere CMEs were separated by CHs from 1 AU footpoints, Kunches and Zwickl (1999) in their sample of 87 SEP events from all solar longitudes found interposed $\mathrm{CHs}$ for only 21 events, all in the eastern hemisphere. Considering this hemispherical difference, the two studies are not strictly incompatible, but it is puzzling that in one study (Shen et al., 2010) most western-hemisphere CMEs had interposed CHs and in the other (Kunches and Zwickl, 1999) none did. This might be the result of using solar He $10830 \AA$ images versus photospheric-field extrapolations for the $\mathrm{CH}$ determinations, but it leaves unresolved the basic question of $\mathrm{CH}$ influence on SEP events.

\section{2. $\mathrm{CH}$ Deflections of Fast CMEs and Possible Effects on SEP Events}

The $\mathrm{CH}-\mathrm{SEP}$ relationship has thus far been considered in the context of a given injection of SEPs at or near the CME source AR. Another view is that the nonradial field of an adjacent $\mathrm{CH}$ may deflect the $\mathrm{CME}$ in a direction away from the $\mathrm{CH}$ location. This has been shown to be the case for interplanetary shocks from CMEs near the central meridian, but without observed accompanying CME drivers (driverless) (Gopalswamy et al., 2009). Studies using a $\mathrm{CH}$ influence vector parameter (CHIP) based on measured properties of observed disk CHs to compare with driverless ICMEs (Gopalswamy et al., 2010), position angles of fastest CME propagation (Mohamed et al., 2012), and presence of magnetic clouds (MCs) (Mäkelä et al., 2013) strongly support the concept of CH deflections of CMEs. This and other evidence of nonradial $\mathrm{CME}$ propagation raises the possibility that $\mathrm{CH}$ deflections of CMEs may lead to modulations of SEP events.

In our previous work (Kahler, Akiyama, and Gopalswamy, 2012, hereafter KAG) we compared the parameters of 41 SEP events with the expected deflections of CMEs originating within $20^{\circ}$ of the central meridian. We not only found no effects of the CME deflections on the onset and rise times and on the peak intensities of SEP events, but also no relationship between these SEP parameters and the initial directions of CME propagations. This suggested that the SEPs may be produced in shock sources much larger than the CMEs. In accord with this possibility, Wood et al. (2012) recently reported an observation by the Solar Terrestrial Relations Observatory/Sun Earth Connection Coronal and Heliospheric Investigation (STEREO/SECCHI) instruments of an eastward deflection of a fast CME by a $\mathrm{CH}$ located on its western flank. The CME-driven shock, however, readily expanded into the adjacent fast stream region of the $\mathrm{CH}$ and was observed in situ more than a day earlier at STEREO-A than at Wind at 1 AU. Although not discussed by Wood et al. (2012), this CME was associated with an $E>10 \mathrm{MeV} 50$ proton flux unit [pfu $=1$ proton $\mathrm{cm}^{-2} \mathrm{~s}^{-1} \mathrm{sr}^{-1}$ ] event observed by the GOES spacecraft. The AR 11164 source of the CME was well connected to Earth at N24W59, so while that event might show that CHs are not impacting SEP events by CME deflections, CHs might still be important for SEP propagation to $1 \mathrm{AU}$, as found by Kunches and Zwickl (1999).

\subsection{SEP Events and SW Streams}

The related question of SEP production by shocks in high-speed streams from $\mathrm{CHs}$ was taken up by Kahler (2004), who argued that two factors mitigate against the production 
of SEPs in high-speed streams. The first is that both the Alfvén and flow speeds of highspeed streams exceed those of the low-speed streams, making it less likely for fast CMEs to drive shocks in the high-speed streams. The second is that if suprathermal ions with speeds extending to $>$ ten times the SW speed are the seed populations of SEP events, those populations are much less intense in the high-speed streams (Gloeckler, 2003). Despite these arguments, Kahler (2004) found not only the presence of SEP events in high-speed streams, but also no requirement for the associated CMEs to be any faster than those with SEP events in low-speed streams. Expanded studies using either $\mathrm{SW} \mathrm{O}^{+7} / \mathrm{O}^{+6}$ values (Kahler, 2005) or the SW stream types (Kahler, 2008) classified by Richardson, Cane, and Cliver (2002) again showed no dependence of SEP event timescales or intensities on SW-stream type.

\subsection{Magnetic Connectivity to CHs and Effects on SEP Events}

The angular separation between the 1 AU footpoints and the source CMEs is assumed to be an important determinant of SEP events. The locations of these footpoints based on simple kinematic extrapolation of 1 AU SW speeds can be misleading because interplanetary field lines invariably converge to CHs (e.g. Luhmann et al., 2009; Wang et al., 2010) that may be substantially displaced in latitude and/or longitude from an assumed W60 (Shen et al., 2006, 2010) or kinematic SW-speed (Kunches and Zwickl, 1999) source. Here we perform another SEP-CH comparison using the Wang-Sheeley-Arge (WSA) model (Arge and Pizzo, 2000; Arge et al., 2004). WSA is a combined empirical and physics-based representation of the corona and quasi-steady global SW flow. The coronal portion of the WSA model is a coupling of the Potential Field Source Surface model (PFSS) and the Schatten Current Sheet (SCS) model with the source surface set to $2.5 \mathrm{R}_{\odot}$ and the SCS model solution used only out to $5 \mathrm{R}_{\odot}$ (hereafter referred to as the outer coronal boundary). The SW portion of the model is a simple 1D modified kinematic model that takes into account stream interactions in an ad-hoc manner. The model propagates SW parcels out to Earth (or any other desired point in space), keeping track of their source regions back at the Sun (i.e. the latitude and longitudes of their photospheric-field footpoints) along with other parameters such as the polarity and field strengths of the footpoints. Hence, each WSA SW prediction at $1 \mathrm{AU}$ comes with a direct mapping of its magnetic-field line back to its $1 \mathrm{AU}$ footpoint at the Sun. Since stream interactions are taken into account in the WSA approach, it is more reliable than the traditional method of mapping the SW back to the Sun assuming a constant speed. We approach the relationship of SEP events and CHs from a more general viewpoint by asking whether the CME location relative to the WSA-based 1 AU footpoint has any bearing on SEP event characteristics.

\section{Data Analysis}

\subsection{Selection of SEP Events and Parameters}

For this work we used the 41 SEP events selected by KAG from Kahler (2013) for their analysis of CME deflections by $\mathrm{CH}$ magnetic fields. The solar sources of the $20 \mathrm{MeV}$ SEP events lay within $20^{\circ}$ of the central meridian, with the basic parameters taken as the peak SEP intensity $I_{\mathrm{p}}$, the SEP onset time $T_{\mathrm{O}}$, defined as the time from CME onset to SEP onset at the Wind spacecraft, and $T_{R}$ : the time from SEP onset to the half maximum of $I_{\mathrm{p}}$. The SEP onset date and event parameters are given in the first four columns of Table 1. The CME source region, determined by the associated flare location, is given in the fifth column. 
出总|

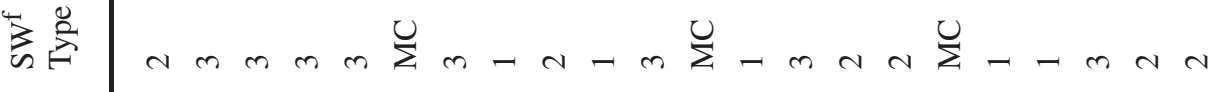

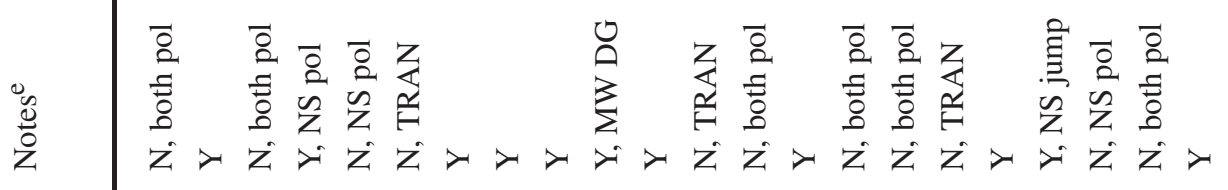

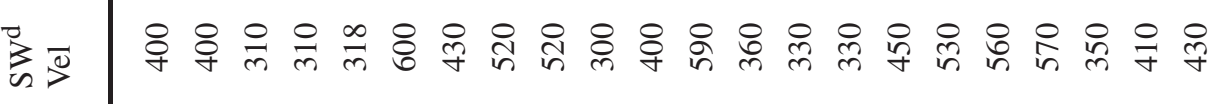
诲

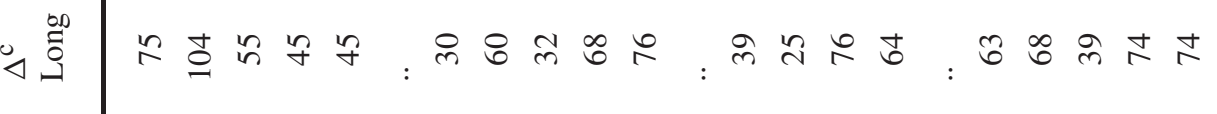

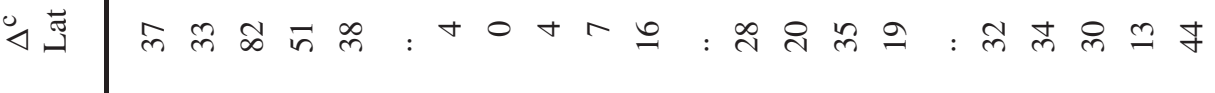

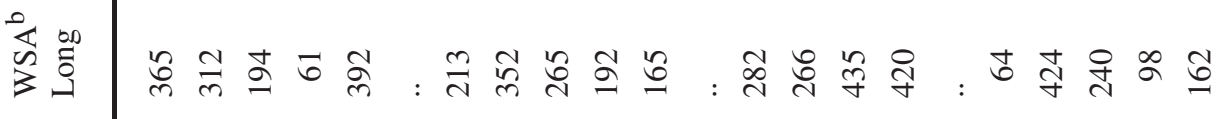

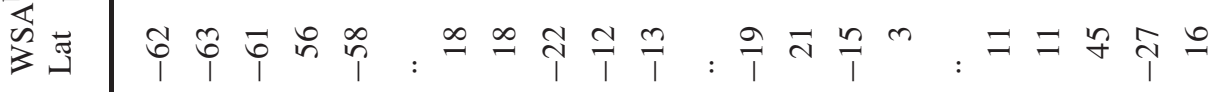




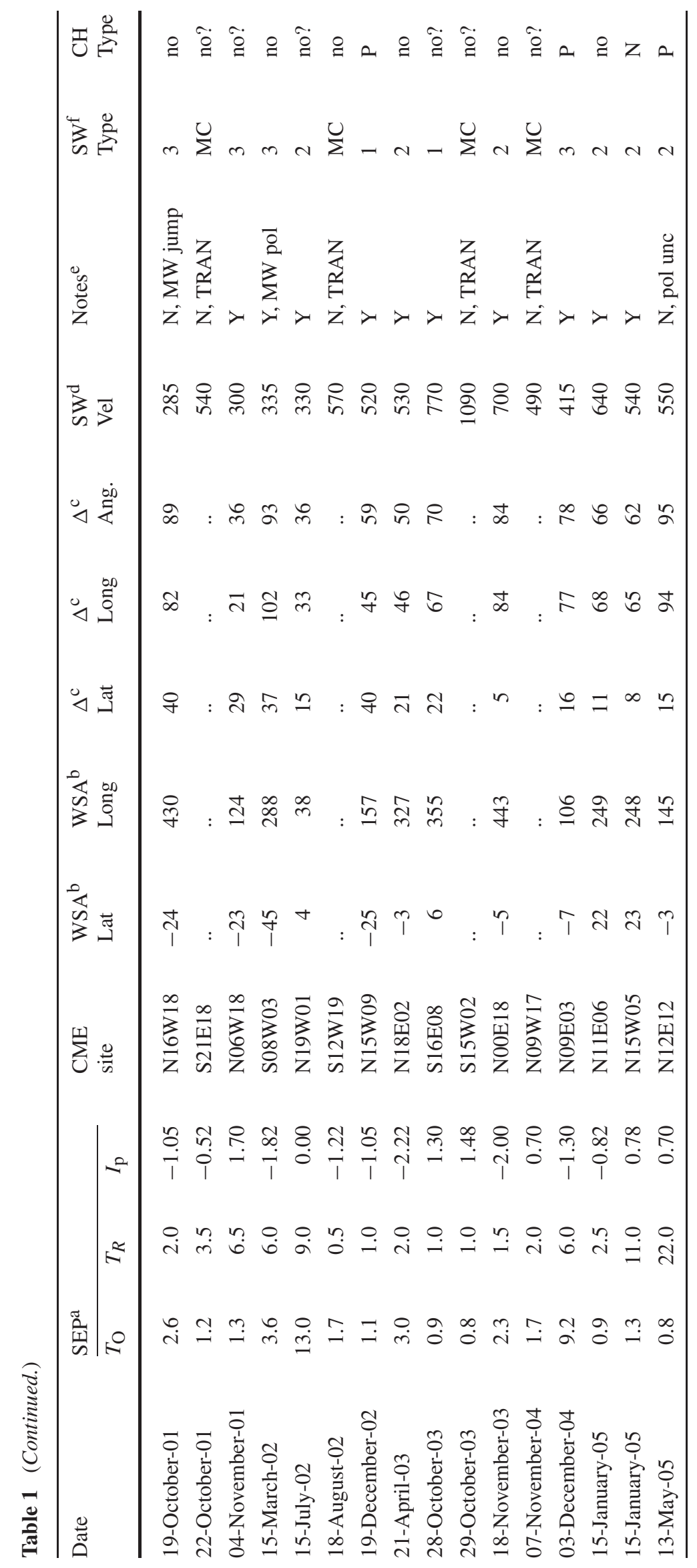




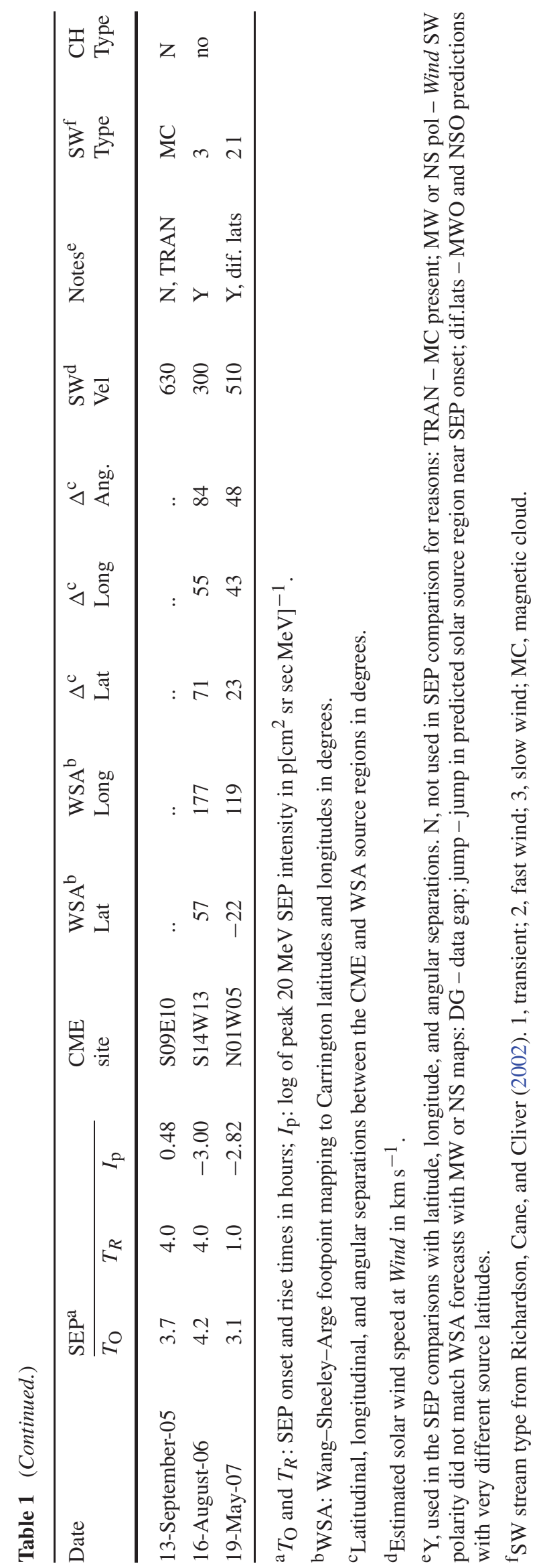



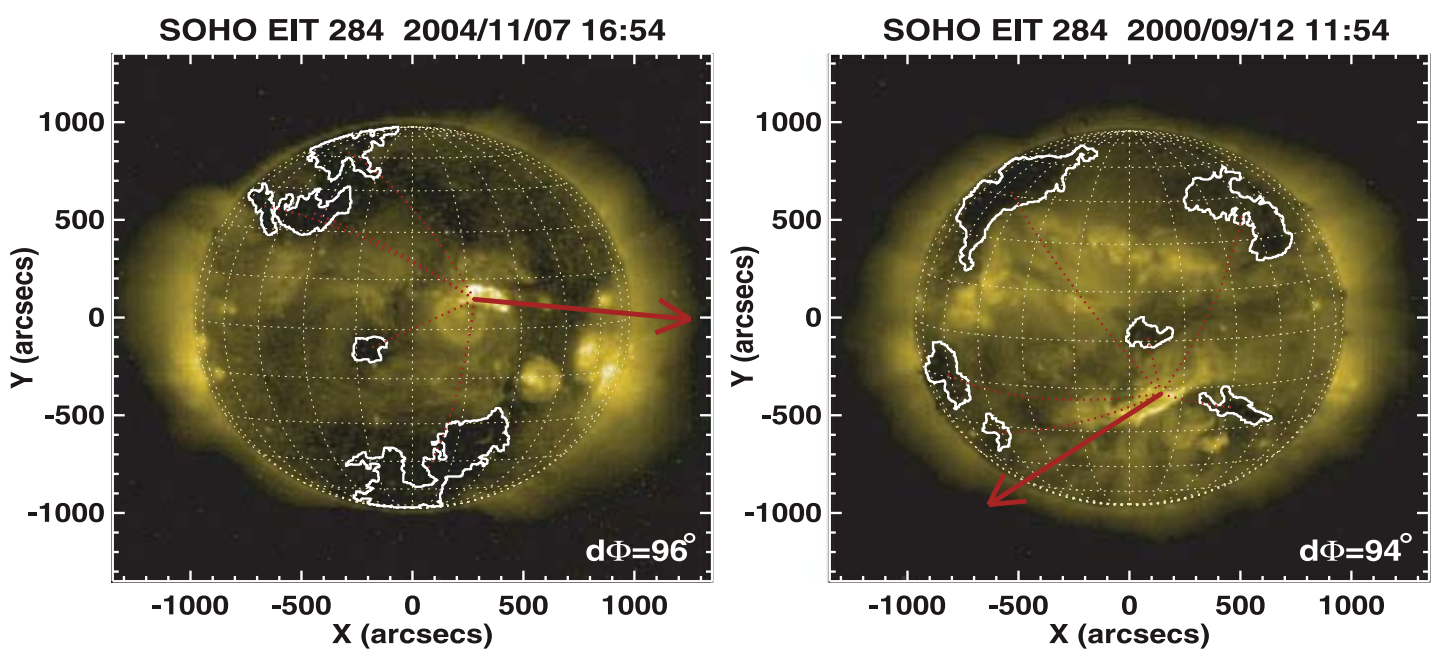

Figure 1 SOHO/EIT $284 \AA$ images used by KAG to determine the $\mathrm{CH}$ properties and boundaries, outlined in white. The red arrows indicate the expected $\mathrm{CME}$ deflections from the $\mathrm{CH}$ source regions at the tails of the arrow. Left: The EIT image of 7 November 2004 without an intervening CH between CME source and the 1 AU footpoint. Right: The EIT image of 12 September 2000 with an intervening negative polarity $\mathrm{CH}$ West of the CME source region.

\subsection{CH Locations and SEP Events}

Our first search for $\mathrm{CH}$ effects on SEP events was to determine whether SEP event parameters are dependent on the presence of CHs located between the central-meridian SEP CME source ARs and the 1 AU footpoints determined from the SW speeds of Table 1. We used the locations of CHs identified by KAG from SOHO/EIT full-disk $284 \AA$ images (Figure 1). This was done without regard to either the $\mathrm{CH}$ size or field intensity, or to the interplanetary magnetic-field configuration during the SEP onset. We ignored high-latitude $\left(>35^{\circ}\right)$ and eastern-hemisphere $\mathrm{CH}$ s to select $\mathrm{CH}$ s only in the same latitude ranges as the $\mathrm{CME}$-source ARs. The $\mathrm{CH}$ configurations are given in the last column of Table 1. A P or N indicates an interposed $\mathrm{CH}$ of positive or negative polarity, such as that shown in the southwest quadrant in the right panel of Figure 1, or no $\mathrm{CH}$ when there is no $\mathrm{CH}$, but with an additional (?) for 11 uncertain cases. In the latter category were $\mathrm{CHs}$ at about the same longitude range as the CME source or those extending only slightly below a latitude of $35^{\circ}$, as in the example in the left panel of Figure 1.

We matched the three SEP parameters of the 11 cases of interposed CHs first with all 30 of the no- $\mathrm{CH}$ cases and then with only the 19 certain cases of no $\mathrm{CH}$. The median values for each case are given in the first part of Table 2: Solar CH Configuration. Differences among these median values are dwarfed by the large standard deviations of the parameters of the full set of 41 events given in the last line of Table 2. Both groups of SEP events, those with and without intervening $\mathrm{CHs}$, display a broad range of overlapping SEP parameters. We show the onset times $T_{\mathrm{O}}$ for the two groups as a function of solar source longitude in Figure 2, which can be compared with Figure 1 of Kunches and Zwickl (1999). The plotted longitude range of these authors extends from $0^{\circ}$ to $\mathrm{E} 90^{\circ}$, but even in the range of $0^{\circ}$ to $\mathrm{E} 20^{\circ}$, common to both plots, their SEP events with $\mathrm{CHs}$ have clearly longer $T_{\mathrm{O}}$ than those without, while our Figure 2 shows no significant difference between the two groups. 
Table 2 Median values of SEP event parameters.

\begin{tabular}{|c|c|c|c|}
\hline SEP/CME or SW & $\log I_{\mathrm{p}}$ & $T_{\mathrm{O}}^{\mathrm{a}}$ & $T_{R}^{\mathrm{a}}$ \\
\hline \multicolumn{4}{|l|}{ Solar CH Configuration } \\
\hline With CH (11 events) & -1.05 & 1.8 & 5.5 \\
\hline All no $\mathrm{CH}$ (30 events) & -1.37 & 2.2 & 2.0 \\
\hline Certain no $\mathrm{CH}$ (19 events) & -1.82 & 2.3 & 2.0 \\
\hline \multicolumn{4}{|l|}{ SW Stream Type } \\
\hline Class 1 (ICME, 15 events) & 0.30 & 1.7 & 2.0 \\
\hline MCs ( 8 events subset) & 0.39 & 1.5 & 2.7 \\
\hline Class 2 (fast SW, 13 events) & -1.52 & 3.0 & 2.0 \\
\hline Class 3 (slow SW, 13 events) & -1.70 & 2.5 & 4.0 \\
\hline All 41 SEP Events & -1.22 & 2.1 & 2.0 \\
\hline Standard Dev (41 events) & 1.42 & 2.9 & 5.4 \\
\hline
\end{tabular}

${ }^{\mathrm{a} O n s e t}$ and rise times in hours.

Figure 2 The SEP onset times $\left[T_{\mathrm{O}}\right]$ as a function of solar source longitude for the $11 \mathrm{SEP}$ events with (squares) and 30 SEP events without (diamonds) CHs lying between the CME source ARs and the assumed 1 AU magnetic footpoints at $\approx \mathrm{W} 60^{\circ}$. Although the number of SEP events with interposed CHs is limited, the distributions of the two groups appear to overlap, which is inconsistent with the idea of a role for $\mathrm{CH}$ in SEP events.

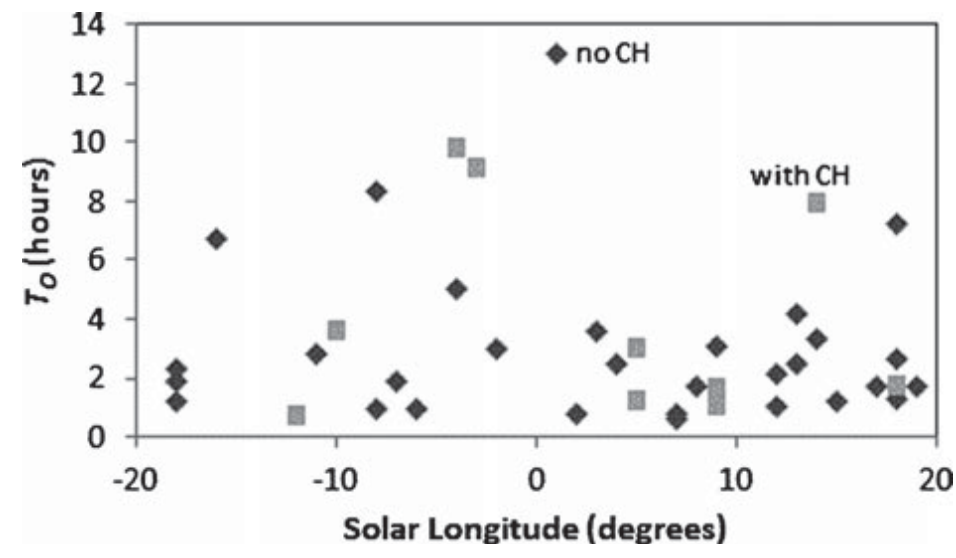

\subsection{Solar Wind Stream Types and SEP Events}

We sorted the SEP event onset times into the three SW stream classes first discussed by Richardson, Cane, and Cliver (2002). These are i) transient structures, including interplanetary CMEs (ICMEs), shocks, and postshock flows; ii) fast SW streams; and iii) slow SW streams. The criteria for ICME selections were updated (Richardson and Cane, 2005) and the revised list of ICME periods posted at www.ssg.sr.unh.edu/mag/ace/ACElists/ICMEtable. html. The three-stream classification periods updated throughout 2008 (I. Richardson, private communication, 2013) were used here. From the SW stream class i) we selected the times of magnetic clouds (MCs) for separate consideration. The distribution of the 41 SEP events was 15 ICMEs, of which 8 were MCs, and 13 events in each of classes ii) and iii). Under SW Stream Type of Table 2 we show the median values of the SEP parameters for each group.

We found slightly lower values of $T_{\mathrm{O}}$ for the ICME group than for the other SW stream groups, consistent with what Kahler (2008) found for his larger group of SEP events, but in view of the large standard deviation of 2.9 hours for all 41 events, the result is not significant. On the other hand, the median $\log I_{\mathrm{p}}$ of 0.30 for the ICME streams is more than a standard deviation above those of the other two SW groups. To visualize that difference, we plot the 
Figure 3 Logs of the peak $20 \mathrm{MeV}$ SEP event intensities $\left[I_{\mathrm{p}}\right]$ versus the parent solar $\mathrm{CME}$ longitudes. No significant difference in $I_{\mathrm{p}}$ is found between events in fast SW regions (blue diamonds) and in slow SW regions (red squares), but SEP events in transient SW regions (green triangles) are somewhat enhanced.

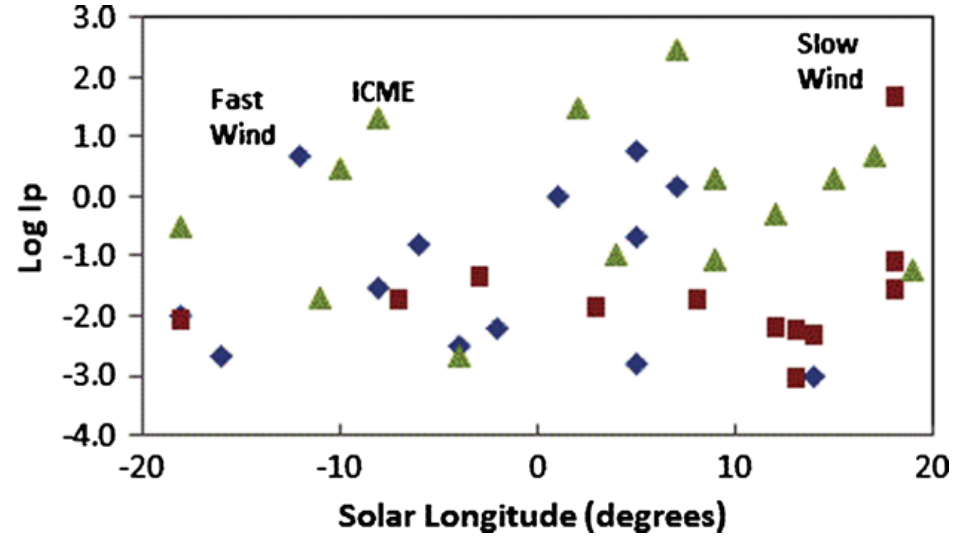

logs of $I_{\mathrm{p}}$ versus solar longitude in Figure 3. Note that three of the eight SEP events in MCs (2 May 1998, 14 July 2000, and 4 November 2001) were ground-level events (GLEs: Reames, 2009). We conclude that there is an indication, still not statistically significant, that SEP events may be more intense in the ICME streams. Returning to our goal of searching for $\mathrm{CH}$ effects via the fast SW streams of group ii), we do not find from Table 2 that these events are distinguished from the SEP events of the other SW groups.

\subsection{WSA Solar Footpoint Connections and SEP Events}

We began with maps of photospheric CHs derived from the WSA model using as inputs to the model Carrington photospheric magnetic-field maps available from Mount Wilson Observatory (MWO) and the National Solar Observatory (NSO). Figure 4 shows two Carrington maps of $\mathrm{CH}$ fields with the connections from a $5 \mathrm{R}_{\odot}$ outer coronal boundary to the photosphere. We generated four-day advance forecasts of 1 AU SW speeds and magneticfield polarities based on the WSA model (Section 1.4) using photospheric magnetic-field observations of both MWO and NSO. The forecast pairs were generated by letting SW parcels leave the Sun at uniform cadence (one parcel for every $2.5^{\circ}$ of solar rotation), but due to their varying departing speeds, they arrive at Earth at nonuniform times, with approximately five predictions per day using $2.5^{\circ}$ resolution maps. Each forecast included not only the 1 AU SW speed and magnetic-field polarity, but also the Carrington longitude and latitude of the $1 \mathrm{AU}$ footpoint. The calculated MWO and NSO footpoints typically agreed to within $5^{\circ}$ to $10^{\circ}$; the averaged source latitudes and longitudes for the SEP events are given in Columns 6 and 7 of Table 1, except for the eight cases when MCs at 1 AU rendered the forecasts moot. In Columns 8 and 9 we give the latitudinal and longitudinal separations between the $33 \mathrm{CME}$ source regions and the WSA 1 AU footpoints, and in Column 10 their angular separations computed from the law of cosines for spherical surfaces (Smart, 1977).

In typical analyses of SEP events (e.g. Kunches and Zwickl, 1999), the 1 AU footpoint is calculated from a simple kinematic extrapolation based on the 1 AU SW speed. We give in Column 11 the SW speeds observed on the Wind spacecraft and obtained from the NASA Coordinated Data Analysis Workshop (CDAW) website. We computed the longitudinal and latitudinal differences between the WSA and kinematic 1 AU footpoints and plot these differences in Figure 5. The large $>50^{\circ}$ latitudinal differences are due to the polar $\mathrm{CH}$ connections during 1997, as shown in the top of Figure 4. Taking the WSA model as definitive, from Figure 5 we see that the characteristic error in determining footpoints by tracking back from SW speeds alone is $\approx 20^{\circ}$ in both latitude and longitude.

The MWO and NSO four-day advance forecasts of SW speeds and magnetic-field polarities were validated in a comparison with values observed at the Wind spacecraft. The 


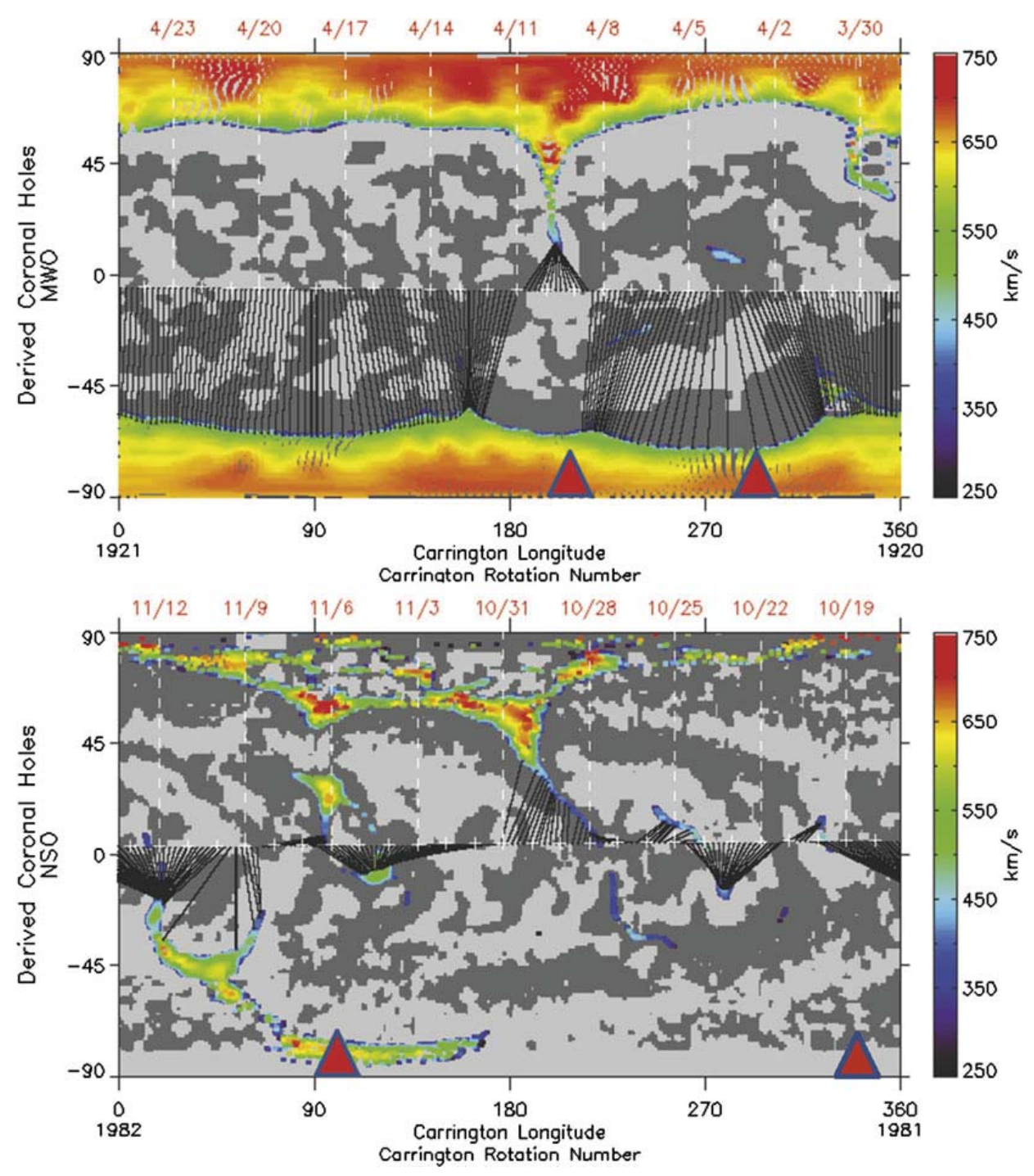

Figure 4 Carrington synoptic maps of derived CHs color-coded for SW speeds. Diagonal lines show the magnetic connections from the sub-Earth points on the $5 \mathrm{R} \odot$ outer coronal boundary to the photospheric $\mathrm{CHs}$ at the dates at the top of each map. Gray areas are closed fields. The footpoint connections at the times of the SEP events correspond to the dates several days preceding the SEP events. Top: the MWO map of CR 1921 showing the $1 \mathrm{AU}$ high-latitude footpoint connections. The CME sources of the SEP events of 1 and 7 April 1997 lie at CR longitudes of $290^{\circ}$ and $208^{\circ}$ (red triangles), respectively, while the modeled 1 AU connections lie at longitudes $346^{\circ}$ and $314^{\circ}$. Bottom: the NSO map of CR 1982 showing the 1 AU lowand intermediate-latitude footpoint connections. The CME sources of the SEP events of 19 October and 4 November 2001 lie at CR longitudes of $348^{\circ}$ and $103^{\circ}$ (red triangles), respectively, and the modeled 1 AU connections lie at longitudes $70^{\circ}$ of the preceding CR 1981 and $124^{\circ}$.

polarity agreements based on both the observed magnetic-field azimuthal directions and the electron heat-flux directions observed with the UC Berkeley 3DP instrument were the primary test of the forecasts, and the SW speed was a secondary consideration. When both the MWO and NSO forecasted polarities disagreed with the 1 AU data, we eliminated the SEP events from the subsequent consideration. These and the MC events were eliminated and listed as N, with notations, in Column 12 of Table 1. Forecasts and 1 AU footpoints of the remaining 23 SEP events were considered validated, listed as $\mathrm{Y}$, and used in the analysis here, although five Y events are qualified with some uncertainty, as noted in Column 12. 
Figure 5 Absolute values of longitudinal and latitudinal differences between the WSA and the SW speed $1 \mathrm{AU}$ footpoints for the 33 non-MC events of Table 1.

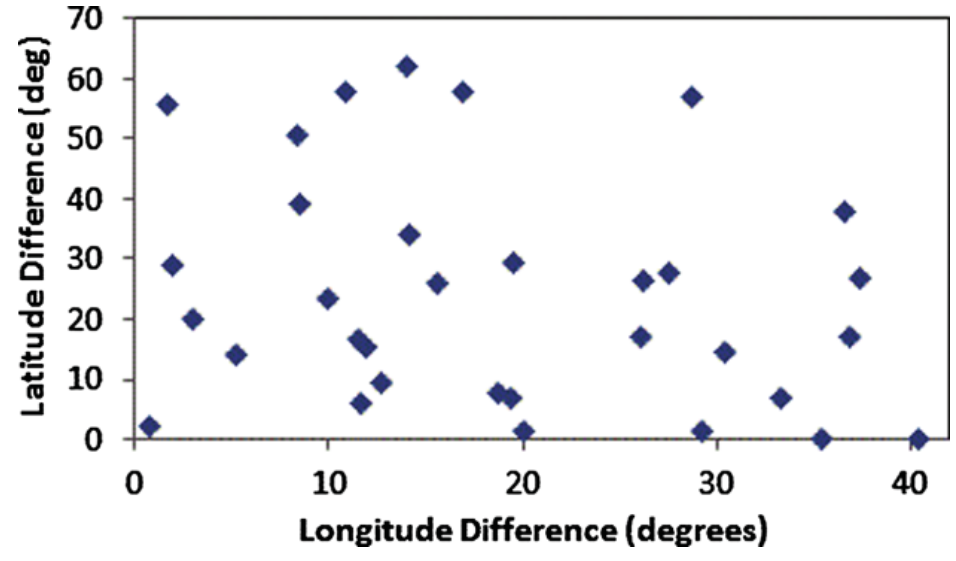

The question that we address here is whether the SEP event parameters depend on the separations of the CME source regions from the $1 \mathrm{AU}$ footpoints of the WSA model. We calculated the correlation coefficients between the three SEP parameters and the latitudinal, longitudinal, and angular separations between the CME sources and 1 AU footpoints and found that in eight of the nine matches the correlation coefficients are $<0.25$, with significance probabilities lower than $80 \%$ for the 23 SEP events. Only $T_{R}$ versus latitudinal separation had a higher correlation coefficient of -0.39 (a decreasing $T_{R}$ with increasing latitudinal separation), still lower than a $95 \%$ significance probability. Since we might have expected the SEP onset time $T_{\mathrm{O}}$ to increase with increasing separation between CME source and 1 AU footpoint, we show latitudinal and longitudinal separations of that parameter for the $23 \mathrm{SEP}$ events in Figure 6 . In both cases these separations ranged up to $\approx 80^{\circ}$, but $T_{\mathrm{O}}$ was not ordered by these parameters. This means that SEP properties do not significantly vary between situations in which the $1 \mathrm{AU}$ footpoints lie in high polar-latitude $\mathrm{CHs}$, as in the example at the top of Figure 4, or in smaller low-latitude CHs shown in the bottom panel of Figure 4.

\section{Summary and Discussion}

\subsection{Null Effects of CH Fields on SEP Events}

SEP events are observed over wide ranges of both latitude (Dalla et al., 2003; Malandraki et al., 2009) and longitude (Cliver et al., 2005) and exhibit broad ranges of $T_{\mathrm{O}}$ and $T_{R}$ as well as peak intensities $I_{\mathrm{p}}$ (Kahler, 2005, 2013), which are currently unexplained. It has long been known that the intensity-time profiles of gradual SEP events are ordered by at least two important factors. The first is the proximity of the source region, which we assumed to be the AR source of a fast and wide CME, to the 1 AU footpoint, typically in the longitudinal range of $\approx \mathrm{W} 40^{\circ},-\mathrm{W} 70^{\circ}$ (e.g. van Hollebeke, Ma Sung, and McDonald, 1975; Cane and Lario, 2006; Reames, 2009; Gardini, Laurenza, and Storini, 2011). The second factor is the propagation of the CME-driven shock (Cane, Reames, and von Rosenvinge, 1988; Reames, Barbier, and Ng, 1996). SEP events may be additionally modulated by closed interplanetary magnetic topologies (Richardson, Cane, and von Rosenvinge, 1991) or through SEP reflections at magnetic-field enhancements that enhance for downstream observer SEP intensities (Kocharov et al., 2009; Tan et al., 2009) and retard or diminish intensities for upstream observers (Lario et al., 2008). Whether a fast and wide CME will even produce an observable SEP event at 1 AU also appears to depend on the CME interaction 
Figure 6 The 23 SEP event onset times $\left[T_{\mathrm{O}}\right]$ versus longitudinal (top) and latitudinal (bottom) separations of the CME source region and the $1 \mathrm{AU}$ footpoint connection. Correlation coefficients were -0.13 and -0.03 for the top and bottom plots.
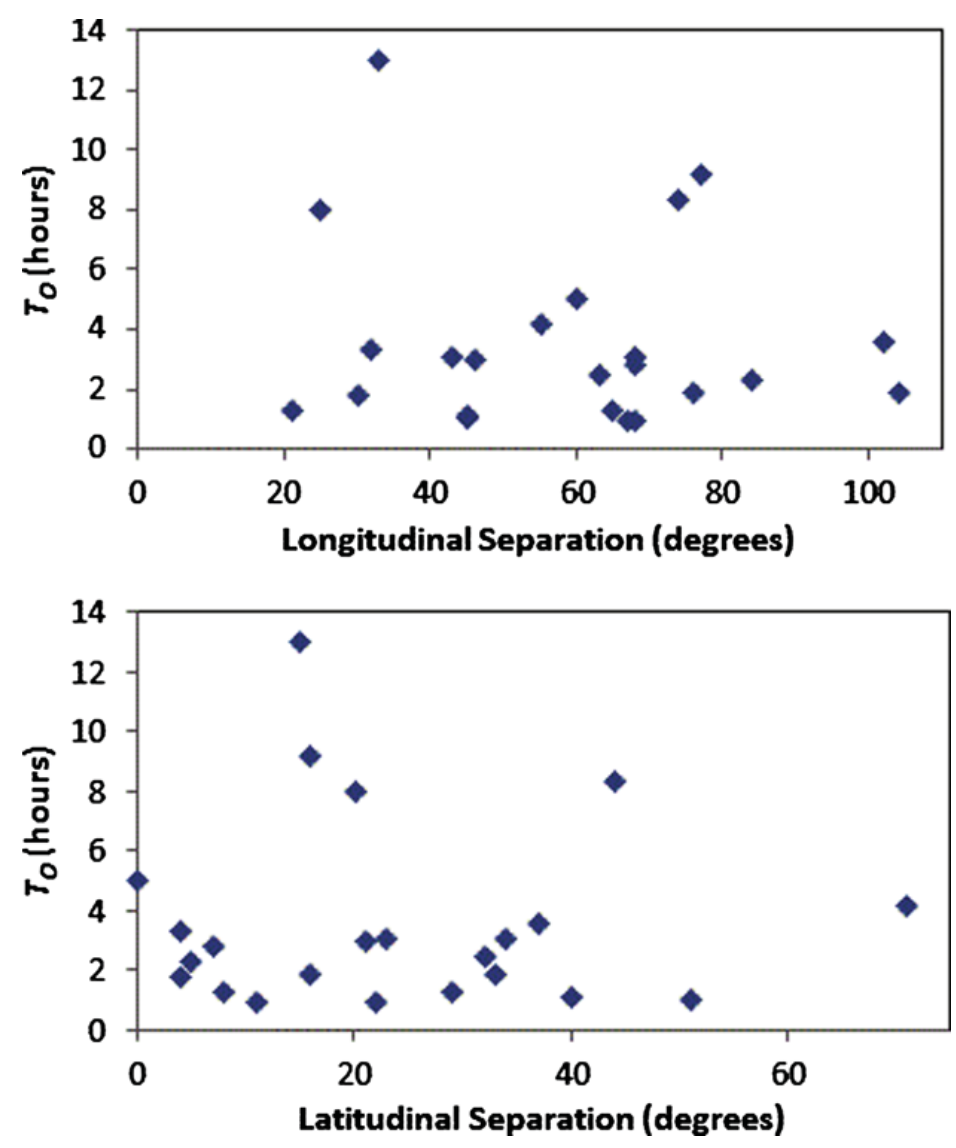

with a streamer or previous CME (Gopalswamy et al., 2003; Kahler and Vourlidas, 2005; Ding et al., 2013). CME widths and speeds correlate weakly with $T_{R}$, although not with $T_{\mathrm{O}}$ (Kahler, 2005, 2013).

We might expect additional organizing factors based on the large-scale coronal and solar structures encountered by the fast and wide CMEs and by their preceding fast shocks that are responsible for producing SEPs. As we discussed in Section 1, CHs have long been suspected to be a factor, although the proposed mechanism of simply blocking or retarding the SEPs released in the solar corona seems simplistic in light of the current paradigm of widespread shock acceleration of SEPs.

The goal of this and the companion work (KAG) has been to search for a statistical effect in the basic time-intensity profiles of SEP events that could be related to CHs. We selected a sample of SEP events with source regions around the central meridian to search for such effects. The relevant associated $\mathrm{CHs}$ were required to be well observed on the solar disk and in locations such that they could affect the SEP propagation to 1 AU footpoint fieldlines. We used three different techniques to search for these possible $\mathrm{CH}$ effects. First, we followed the approach of Kunches and Zwickl (1999) to divide SEP events into two groups depending on whether a $\mathrm{CH}$ appeared to lie on a line connecting the SEP source and the 1 AU footpoint. This simple concept belies the complexity of determining i) the SEP source region, which involves the large-scale $\mathrm{CME}$, ii) the extent of the $\mathrm{CH}$ field lines, which extend nonradially from their apparent wavelength-dependent source boundaries, and iii) the $1 \mathrm{AU}$ footpoints, which generally lie tens of degrees in latitude and longitude away from their kinematically computed sources (Figure 5). Contrary to Kunches and Zwickl (1999), we did not find $T_{\mathrm{O}}$ delayed for SEP events with interposed $\mathrm{CHs}$ (Figure 2 ) in the E20 $0^{\circ}-0^{\circ}$ longitude regions common to the two studies. We do not understand (Section 1.2) why they found no 
intervening $\mathrm{CH}$ s for their western-hemisphere SEP events, but their stricter requirement that a $\mathrm{CH}$ must lie on a line connecting the SEP and $1 \mathrm{AU}$ footpoint regions, as opposed to lying in an intermediate longitude, may be a factor. A previous comparison of He 10830 $\AA$ used by Kunches and Zwickl (1999) and soft X-ray CH boundaries (Kahler, Davis, and Harvey, 1983) showed poor agreement for low-latitude $\mathrm{CH}$ boundaries. Our results, that peak intensities $\left[I_{\mathrm{p}}\right]$ are not dependent on $\mathrm{CH}$ locations, agree with those of Shen et al. (2006).

Comparisons of SEP event properties in different types of SW streams were our second approach in searching for evidence of $\mathrm{CH}$ effects, this time through their associated high-speed streams. While we did find higher SEP $I_{\mathrm{p}}$ values among the ICME MC group of events, including three GLEs, SEP events of the fast SW group ii) streams were not distinguished from the others in terms of their SEP events (Table 2). This result is consistent with the lack of any significant variation of SEP event characteristics with SW stream type in the broader survey of Kahler (2008). The additional lack of a significant SW stream variation in SEP elemental composition (Kahler, Tylka, and Reames, 2009) supports the conclusion that the SW stream structure is simply not a determining factor for SEP propagation.

Since the work of Kunches and Zwickl (1999), we recognize that the 1 AU footpoints invariably trace the edges or interiors of $\mathrm{CHs}$, which may be far removed from the nominal 1 AU ecliptic projections. Although the PFSS model accuracy may be limited (Nitta and De Rosa, 2008), a footpoint connection to the $\mathrm{CH}$ vicinity of a flaring AR is crucial for observing impulsive SEP events (Rust et al., 2008) and for determining the seed-particle population for shock-accelerated events (Ko et al., 2012). We aimed at a more accurate comparison between the CME source regions and the $1 \mathrm{AU}$ footpoints (Figure 4). We searched for effects on SEP events that could be attributed to variations of these footpoint locations by sorting the SEP events on the basis of the angular separations between their source regions and the calculated 1 AU footpoints. We interpreted the null result of Figure 6 as an indication that the low coronal magnetic-field connection does not order SEP events, perhaps because the shock propagation and SEP injection are occurring above the outer coronal boundary $5 \mathrm{R}_{\odot}$ source height where the PFSS fields are presumed to be nearly radial. Our study has been confined to SEP events originating within a $40^{\circ}$ band of the central meridian, so the possibility of $\mathrm{CH}$ effects on SEPs from other regions, particularly eastern-hemisphere sources (Kunches and Zwickl, 1999), cannot be ruled out. We did not attempt to include elemental composition as an SEP event property, but other studies give no indication that stream structures play a role in SEP composition (Kahler, Tylka, and Reames, 2009, 2011).

\subsection{SEP Events and Latitudinal Separations of 1 AU Footpoints}

The latitudinal separations between associated AR flares and the 1 AU footpoints rarely exceed about $30^{\circ}$ for SEP events and are generally ignored in comparison with the much wider range of longitudinal separations. However, the observation of Ulysses high-latitude SEP events prompted a new search for possible latitude effects on SEP event characteristics. Dalla et al. (2003) found that $E \approx 30 \mathrm{MeV}$ SEP event times to maximum, roughly equivalent to our $T_{\mathrm{O}}+T_{R}$, increased with increasing latitudinal separations for nine Ulysses highlatitude events. For the corresponding nine SEP events observed at $1 \mathrm{AU}$, however, their SEP times to maxima showed no latitudinal dependence, similar to our Figure 6. Dalla and Agueda (2010) followed this Ulysses 1 AU comparison with a larger 1 AU study based on the nominal latitudinal separations $\Delta \theta$ of 496 well-connected $\geq \mathrm{C} 8$ solar flares. The probability of detecting any associated SEP event at 1 AU peaked in the range of $\Delta \Theta=$ $4^{\circ}-12^{\circ}$, but as in the Dalla et al. (2003) study, the SEP times to maximum showed no 
dependence on $\Delta \Theta$, suggesting that it is the larger-scale interplanetary and not the coronal latitudinal and longitudinal field-line separations that are important for SEP propagation. This conclusion is supported by modeling of SEP profiles for different latitudinal separations between the observer and the progenitor CME (Rodríguez-Gasén et al., 2011). This result may not apply at the highest energies of ground-level enhancement (GLE) events, however. Gopalswamy et al. (2013) found that while fast CMEs from well-connected solar longitude regions produced strong SEP events in Solar Cycle 24, it was necessary for the CME nose to be close $\left(\leq 5^{\circ}\right)$ to the ecliptic plane to produce a GLE. Otherwise, only lower-energy SEPs, presumably from the shock flanks, reached Earth.

Since large-scale coronal and interplanetary structures seem to give only rough guidance to SEP event timescales, the strong variability of SEP event intensities and timescales observed at $1 \mathrm{AU}$ may be due to spatial and temporal variations inherent in the shocks themselves (Kóta, 2010) and to their time-dependent connections to the field lines at $1 \mathrm{AU}$. We may expect better understanding of SEP profiles from more detailed modeling efforts based on multiple-imaging observations of interplanetary shock fronts and SEPs, such as those of Rouillard et al. (2011).

Acknowledgements SWK was funded by AFOSR Task 2301RDZ4. NG and SA were supported by NASA's LWS TR\&T program. CME data were taken from the CDAW LASCO catalog. This CME catalog is generated and maintained at the CDAW Data Center by NASA and The Catholic University of America in cooperation with the Naval Research Laboratory. SOHO is a project of international cooperation between ESA and NASA. EIT images of Figure 1 were obtained from the EIT instrument webpage. We thank Ian Richardson for providing the SW stream listings and Don Reames for the use of the EPACT proton data. We used Wind data provided by J.H. King, N. Papatashvilli, and R. Lepping at the NASA/GSFC CDAW website.

\section{References}

Arge, C.N., Pizzo, V.J.: 2000, Improvement in the prediction of solar wind conditions using near-real time solar magnetic field updates. J. Geophys. Res. 105, 10465 - 10480. doi:10.1029/1999JA900262.

Arge, C.N., Luhmann, J.G., Odstrcil, D., Schrijver, C.J., Li, Y.: 2004, Stream structure and coronal sources of the solar wind during the May 12th, 1997 CME. J. Atmos. Solar-Terr. Phys. 66, 1295-1309. doi:10.1016/j.jastp.2004.03.018.

Bravo, S.: 1993, The SC event of 6 June 1979 and related solar and interplanetary observations. Adv. Space Res. 13, 371 - 374. doi:10.1016/0273-1177(93)90508-9.

Bravo, S.: 1995, A solar scenario for the associated occurrence of flares, eruptive prominences, coronal mass ejections, coronal holes, and interplanetary shocks. Solar Phys. 161, 57 - 65. doi:10.1007/BF00732084.

Cane, H.V., Lario, D.: 2006, An introduction to CMEs and energetic particles. Space Sci. Rev. 123, 45 - 56. doi:10.1007/s11214-006-9011-3.

Cane, H.V., Reames, D.V., von Rosenvinge, T.T.: 1988, The role of interplanetary shocks in the longitude distribution of solar energetic particles. J. Geophys. Res. 93, 9555-9567. doi:10.1029/JA093iA09p09555.

Cliver, E.W., Thompson, B.J., Lawrence, G.R., Zhukov, A.N., Tylka, A.J., Dietrich, W.F., Reames, D.V., Reiner, M.J., MacDowall, R.J., Kosovichev, A.G., Ling, A.G.: 2005, The solar energetic particle event of 16 August 2001: $400 \mathrm{MeV}$ protons following an eruption at $\approx$ W180. In: Acharya, B.S., Gupta, S., Jagadeesan, P., Jain, A., Karthikeyan, S., Morris, S., Tonwar, S. (eds.) Proc. 29th Int. Cosmic Ray Conf. 1, Tata Inst. Fund. Res., $121-124$.

Dalla, S., Agueda, N.: 2010, Role of latitude of source region in solar energetic particle events. In: Maksimovic, M., Issautier, K., Meyer-Vernet, N., Moncuquet, M., Pantellini, F. (eds.) Proc. 12th Int. Solar Wind Conf. CP-1216, AIP, New York, 613-616. doi:10.1063/1.3395941.

Dalla, S., Balogh, A., Krucker, S., Posner, A., Müller-Mellin, R., Anglin, J.D., Hofer, M.Y., Marsden, R.G., Sanderson, T.R., Tranquille, C., Heber, B., Zhang, M., McKibben, R.B.: 2003, Properties of high heliolatitude solar energetic particle events and constraints on models of acceleration and propagation. Geophys. Res. Lett. 30, 8035. doi:10.1029/2003GL017139. ULY 9-1.

Ding, L., Jiang, Y., Zhao, L., Li, G.: 2013, The "twin-CME" scenario and large solar energetic particle events in solar cycle 23. Astrophys. J. 763, 30. doi:10.1088/0004-637X/763/1/30. 
Gardini, A., Laurenza, M., Storini, M.: 2011, SEP events and multi-spacecraft observations: constraints on theory. Adv. Space Res. 47, 2127 - 2139. doi:10.1016/j.asr.2011.01.025.

Gloeckler, G.: 2003, Ubiquitous suprathermal tails on the solar wind and pickup ion distributions. In: Velli, M., Bruno, R., Malara, F. (eds.) Proc. 10th Int. Solar Wind Conf. CP-679, AIP, New York, 583-588. doi:10.1063/1.1618663.

Gopalswamy, N., Yashiro, S., Michalek, G., Kaiser, M.L., Howard, R.A., Leske, R., von Rosenvinge, T., Reames, D.V.: 2003, Effect of CME interactions on the production of solar energetic particles. In: Velli, M., Bruno, R., Malara, F. (eds.) Proc. 10th Int. Solar Wind Conf. CP-679, AIP, New York, 608-611. doi:10.1063/1.1618668.

Gopalswamy, N., Mäkelä, P., Xie, H., Akiyama, S., Yashiro, S.: 2009, CME interactions with coronal holes and their interplanetary consequences. J. Geophys. Res. 114, A00A22. doi:10.1029/2008JA013686.

Gopalswamy, N., Mäkelä, P., Xie, H., Akiyama, S., Yashiro, S.: 2010, Solar sources of “driverless" interplanetary shocks. In: Maksimovic, M., et al. (eds.) Proc. Twelfth Int. Solar Wind Conf. CP-1216, $452-455$. doi:10.1063/1.3395902.

Gopalswamy, N., Xie, H., Akiyama, S., Yashiro, S., Usoskin, I.G., Davilla, J.M.: 2013, The first ground level enhancement of solar cycle 24: direct observation of shock formation and particle release heights. Astrophys. J. 765, L30. doi:10.1088/2041-8205/765/2/L30.

Howard, R.A., Sheeley, N.R. Jr., Michels, D.J., Koomen, M.J.: 1985, Coronal mass ejections - 1979-1981. J. Geophys. Res. 90, 8173-8191. doi:10.1029/JA090iA09p08173.

Kahler, S.W.: 2004, Solar fast-wind regions as sources of shock energetic particle production. Astrophys. J. 603, 330 - 334. doi:10.1086/381358.

Kahler, S.W.: 2005, Characteristic times of gradual solar energetic particle events and their dependence on associated coronal mass ejection properties. Astrophys. J. 628, 1014-1022. doi:10.1086/431194.

Kahler, S.W.: 2008, Time scales of solar energetic particle events and solar wind stream types. In: Caballero, R., D’Olivo, J.C., Medina-Tanco, G., Nellen, L., Sánchez, F.A., Valdés-Galicia, J.F. (eds.) Proc. 30th Int. Cosmic Ray Conf. 1, Univ. Nac. Auto. de Mexico, Mexico, 143-146.

Kahler, S.W.: 2013, A comparison of solar energetic particle event timescales with properties of associated coronal mass ejections. Astrophys. J. 769, 110. doi:10.1088/0004-637X/769/2/110.

Kahler, S.W., Akiyama, S., Gopalswamy, N.: 2012, Deflections of fast coronal mass ejections and the properties of associated solar energetic particle events. Astrophys. J. 754, 100. doi:10.1088/0004-637X/ 754/2/100 (KAG).

Kahler, S.W., Davis, J.M., Harvey, J.W.: 1983, Comparison of coronal holes observed in soft X-ray and He I 10830 A spectroheliograms. Solar Phys. 87, 47 - 56. doi:10.1007/BF00151159.

Kahler, S.W., Kunches, J., Smith, D.F.: 1995, Coronal and interplanetary magnetic sector structure and the modulation of solar energetic particle events. In: 24th Int. Cosmic Ray Conf. 4, 325 - 328.

Kahler, S.W., Kunches, J.M., Smith, D.F.: 1996, Role of current sheets in the modulation of solar energetic particle events. J. Geophys. Res. 101, 24383 -24392. doi:10.1029/96JA02446.

Kahler, S.W., Tylka, A.J., Reames, D.V.: 2009, A comparison of elemental abundance ratios in SEP events in fast and slow solar wind regions. Astrophys. J. 701, 561. doi:10.1088/0004-637X/701/1/561.

Kahler, S.W., Vourlidas, A.: 2005, Fast coronal mass ejection environments and the production of solar energetic particle events. J. Geophys. Res. 110, A12S01. doi:10.1029/2005JA011073.

Kahler, S.W., Cliver, E.W., Tylka, A.J., Dietrich, W.F.: 2011, A comparison of ground level event e/p and Fe/O ratios with associated solar flare and CME characteristics. Space Sci. Rev. 171, 121-139. doi:10.1007/s11214-011-9768-x.

Ko, Y.-K., Tylka, A.J., Ng, C.K., Wang, Y.-M.: 2012, On the relationship between heavy-ion composition variability in gradual SEP events and the associated IMF source regions. In: Hu, Q., Li, G., Zank, G.P., Ao, X., Verkhoglyadova, O., Adams, J.H. (eds.) Space Weather: The Space Radiation Environment 1500, AIP, New York, 26-31. doi:10.1063/1.4768740.

Kocharov, L., Laitinen, T., Al-Sawad, A., Saloniemi, O., Valtonen, E., Reiner, M.J.: 2009, Gradual solar energetic particle event associated with a decelerating shock wave. Astrophys. J. Lett. 700, L51 - L55. doi:10.1088/0004-637X/700/1/L51.

Kóta, J.: 2010, Particle acceleration at near-perpendicular shocks: the role of field-line topology. Astrophys. J. 723, 393 - 397. doi:10.1088/0004-637X/723/1/393.

Kunches, J.M., Zwickl, R.D.: 1999, The effects of coronal holes on the propagation of solar energetic protons. Radiat. Meas. 30, $281-286$.

Lario, D., Decker, R.B., Malandraki, O.E., Lanzerotti, L.J.: 2008, Influence of large-scale interplanetary structures on energetic particle propagation: September 2004 event at Ulysses and ACE. J. Geophys. Res. 113, A03105. doi:10.1029/2007JA012721.

Luhmann, J.G., Lee, C.O., Li, Y., Arge, C.N., Galvin, A.B., Simunac, K., Russell, C.T., Howard, R.A., Petrie, G.: 2009, Solar wind sources in the late declining phase of cycle 23: effects of the weak solar polar field on high speed streams. Solar Phys. 256, 285 - 305. doi:10.1007/s11207-009-9354-5. 
Mäkelä, P., Gopalswamy, N., Xie, H., Mohamed, A.A., Akiyama, S., Yashiro, S.: 2013, Coronal hole influence on the observed structure of interplanetary CMEs. Solar Phys. 284, 59-75. doi:10.1007/s11207-012-0211-6.

Malandraki, O.E., Marsden, R.G., Lario, D., Tranquille, C., Heber, B., Mewaldt, R.A., Cohen, C.M.S., Lanzerotti, L.J., Forsyth, R.J., Elliott, H.A., Vogiatzis, I.I., Geranios, A.: 2009, Energetic particle observations and propagation in the three-dimensional heliosphere during the 2006 December events. Astrophys. J. 704, 469 - 476. doi:10.1088/0004-637X/704/1/469.

Mohamed, A.A., Gopalswamy, N., Yashiro, N., Akiyama, S., Mäkelä, P., Xie, H., Jung, H.: 2012, The relation between coronal holes and coronal mass ejections during the rise, maximum, and declining phases of solar cycle 23. J. Geophys. Res. 117, A01103. doi:10.1029/2011JA016589.

Nitta, N.V., De Rosa, M.L.: 2008, A comparison of solar open field regions found by type III radio bursts and the potential field source surface model. Astrophys. J. Lett. 673, L207 - L210. doi:10.1086/527548.

Reames, D.V.: 2009, Solar energetic-particle release times in historic ground-level events. Astrophys. J. 706, 844 - 850. doi:10.1088/0004-637X/706/1/844.

Reames, D.V., Barbier, L.M., Ng, C.K.: 1996, The spatial distribution of particles accelerated by coronal mass ejection-driven shocks. Astrophys. J. 466, 473 - 486. doi:10.1086/177525.

Richardson, I.G., Cane, H.V.: 2005, A survey of interplanetary coronal mass ejections in the near-Earth solar wind during 1996-2005. In: Fleck, B., Zurbuchen, T.H., Lacoste, H. (eds.) Proc. Solar Wind 11/SOHO 16 Conf. SP-592, ESA, Noordwijk, $755-758$.

Richardson, I.G., Cane, H.V., Cliver, E.W.: 2002, Sources of geomagnetic activity during nearly three solar cycles (1972 - 2000). J. Geophys. Res. 107, SSH 8-1. doi:10.1029/2001JA000504.

Richardson, I.G., Cane, H.V., von Rosenvinge, T.T.: 1991, Prompt arrival of solar energetic particles from far eastern events - the role of large-scale interplanetary magnetic field structure. J. Geophys. Res. 96, 7853 - 7860. doi:10.1029/91JA00379.

Rodríguez-Gasén, R., Aran, A., Sanahuja, B., Jacobs, C., Poedts, S.: 2011, Why should the latitude of the observer be considered when modeling gradual proton events? An insight using the concept of cobpoint. Adv. Space Res. 47, 2140-2151. 10.1016/j.asr.2010.03.021.

Rouillard, A.P., Odstrcil, D., Sheeley, N.R., Tylka, A., Vourlidas, A., Mason, G., Wu, C.-C., Savani, N.P., Wood, B.E., Ng, C.K., Stenborg, G., Szabo, A., St. Cyr, O.C.: 2011, Interpreting the properties of solar energetic particle events by using combined imaging and modeling of interplanetary shocks. Astrophys. J. 735, 7. doi:10.1088/0004-637X/735/1/7.

Rust, D.M., Haggerty, D.K., Georgoulis, M.K., Sheeley, N.R., Wang, Y.-M., De Rosa, M.L., Schrijver, C.J.: 2008, On the solar origins of open magnetic fields in the heliosphere. Astrophys. J. 687, 635-645. doi:10.1086/592017.

Shen, C., Wang, Y., Ye, P., Wang, S.: 2006, Is there any evident effect of coronal holes on gradual solar energetic particle events? Astrophys. J. 639, 510-515. doi:10.1086/499199.

Shen, C.-L., Yao, J., Wang, Y.-M., Ye, P.-Z., Zhao, X.-P., Wang, S.: 2010, Influence of coronal holes on CMEs in causing SEP events. Res. Astron. Astrophys. 10, 1049-1060. doi:10.1088/1674-4527/10/10/008.

Smart, W.M.: 1977, Textbook on Spherical Astronomy, Cambridge Univ. Press, Cambridge.

Tan, L.C., Reames, D.V., Ng, C.K., Saloniemi, O., Wang, L.: 2009, Observational evidence on the presence of an outer reflecting boundary in solar energetic particle events. Astrophys. J. 701, 1753-1764. doi:10.1088/0004-637X/701/2/1753.

van Hollebeke, M.A.I., Ma Sung, L.S., McDonald, F.B.: 1975, The variation of solar proton energy spectra and size distribution with heliolongitude. Solar Phys. 41, 189-223. doi:10.1007/BF00152967.

von Rosenvinge, T.T., Reames, D.V.: 1983, The delayed energetic particle event of June 6-10, 1979. In: Durgaprasad, N., Ramadurai, S., Ramana Murthy, P.V., Rao, M.V.S., Sivaprasad, K. (eds.) 18th Int. Cosmic Ray Conf. 10, Tata Inst. Fund. Res., 373-376.

Wang, Y.-M., Robbrecht, E., Rouillard, A.P., Sheeley, N.R. Jr., Thernisien, A.F.R.: 2010, Formation and evolution of coronal holes following the emergence of active regions. Astrophys. J. 715, 39-50. doi:10.1088/0004-637X/715/1/39.

Wood, B.E., Wu, C.-C., Rouillard, A.P., Howard, R.A., Socker, D.G.: 2012, A coronal hole's effects on coronal mass ejection shock morphology in the inner heliosphere. Astrophys. J. 755, 43. doi:10.1088/0004-637X/755/1/43. 